Research Journal of Biological Sciences 6(10): 507-511, 2011

ISSN: $1815-8846$

(C) Medwell Journals, 2011

\title{
Effect of Simvastatin on PTZ-Induced Seizure in Mice
}

\author{
Khayatnouri Mirhadi \\ Department of Pharmacology, Tabriz Branch, Islamic Azad University, Tabriz, Iran
}

\begin{abstract}
The 3-Hydroxy-3-Methylglutaryl-CoA (HMG-CoA) reductase inhibitors (statins) have been unequivocally shown to reduce cardiovascular morbidity and mortality by their lipid-lowering actions. It has been suggested that statins appear to have therapeutic benefits in diseases that are unrelated to elevated serum cholesterol levels. The aim of this study was to evaluation the effect of intraperitoneally injection of different doses of simvastatin on PTZ-induced seizure threshold in mice. Mice were divided into 9 groups randomly: the 1st group received saline normal (ip) (control group); the 2nd group received Carboxymethylcellulose (CMC) $0.5 \%$ (ip) (vehicle group) and the next groups received, respectively different doses of simvastatin $(1,5,10,20$, 40,80 and $100 \mathrm{mg} \mathrm{kg}^{-1}$ daily) for 4 days (ip) before determination of seizure threshold induced by PTZ. Results showed that PTZ-induced seizure threshold in control mice was $35.48 \pm 1.39 \mathrm{mg} \mathrm{kg}^{-1}$. Intraperitoneal injection of simvastatin showed significant $(\mathrm{p}<0.05)$ increase of PTZ-induced seizure threshold in a dose dependently manner. According to the results, simvastatin has anticonvulsant effects on mice. Nevertheless, new studies must be carried out in order to determine the beneficial effects of statins in treatment of epilepsy.
\end{abstract}

Key words: Simvastatin, seizure, PTZ, threshold, mice, Iran

\section{INTRODUCTION}

Epilepsy is one of the major neurological diseases in humans and about $1 \%$ of the population is involved. The 3-Hydroxy-3-Methylglutaryl-CoA (HMG-CoA) reductase inhibitors (statins) have been unequivocally shown to reduce cardiovascular morbidity and mortality (Scandinavian Simvastatin Survival Study, 1994; Shepherd et al., 1995). Statins are among the most widely used prescription drugs and exert their lipid-lowering actions by reversible and competitive inhibition of the enzyme 3-Hydroxy-3-Methyl-Glutaryl-CoA reductase ( $\mathrm{HMG}-\mathrm{CoA}$ reductase), the rate-limiting step in the conversion of 3-Hydroxy-3-Methyl-Glutaryl-Coenzyme A ( $\mathrm{HMG}-\mathrm{CoA}$ ) to mevalonate, a precursor of cholesterol (McTaggart et al., 2001). It has been suggested that statins appear to have therapeutic benefits in diseases that are unrelated to elevated serum cholesterol levels such as rheumatologic diseases and ischemic stroke (McCarey et al., 2004).

Statins are also known for their pleiotropic effects which are independent of their lipid-lowering properties. Among the effects of statins, the most relevant are anti-atherosclerotic and anti-inflammatory actions, improvement of endothelial dysfunction, anti-thrombosis and anti-oxidant actions, prevention of Alzheimer's disease and antineoplasic actions (Goncalves et al., 2011). Statins might exert beneficial effects beyond cholesterol reduction include improving endothelial function, decreasing vascular inflammation inhibiting smooth- muscle proliferation and immunomodulation. Most of these effects are mediated through inhibition of isoprenoid synthesis with subsequent effects on multiple downstream signaling pathways (Wang et al., 2008). Also, statins might exert beneficial neuroprotective effects in various animal disease models and clinical studies (Daimona et al., 2004; Lee et al., 2008; Rangel et al., 2005). Furthermore, recent studies have shown that statins reduces the extent of brain damage after ischemic insult (Sironi et al., 2003). Therefore, the aim of this study was to evaluation the effect of intraperitoneally injection of different doses of simvastatin on PTZ-induced seizure threshold in mice.

\section{MATERIALS AND METHODS}

Animals: Experiments were performed on 22-25 g adult NMRI male mice in their $8-9$ weeks ( $\mathrm{n}=8$ for each group), purchased from Razi Institute (Iran). Animals were housed 8 per cage in the Animal House of Veterinary Faculty of Tabriz Branch, Islamic Azad University in a temperature $\left(20-22^{\circ} \mathrm{C}\right)$ and humidity $(50 \pm 10 \%)$ controlled environment under a $12 \mathrm{~h} \mathrm{light/dark} \mathrm{cycle} \mathrm{(lights} \mathrm{on} \mathrm{at} 7$ a.m.). Food and water were available ad libitum.

This study was performed in accordance with the Guide for the Care and Use of Laboratory Animals of Research affairs of Tabriz University of Medical Sciences, Tabriz, Iran. All efforts were made to minimize the number of animals which were used and their suffering degree. Animals were divided into 9 groups randomly: the 1 st 
group received saline normal (ip) (control group); the 2nd group received Carboxymethylcellulose (CMC) $0.5 \%$ (ip) (vehicle group) and the next groups received, respectively different doses of simvastatin $(1,5,10,20,40,80$ and $100 \mathrm{mg} \mathrm{kg}^{-1}$ daily) for 4 days (ip) before PTZ-induced seizure threshold.

Chemicals: PTZ (Pentylenetetrazole) and simvastatin was provided from Sigma-Aldrich. All other reagents were of analytical grade. Simvastatin was prepared by being dissolved in $0.5 \%$ Carboxymethylcellulose (CMC) and the vehicle group was given an equal volume of vehicle.

PTZ-induced clonic seizure threshold: Behavioral experiments were done in a quiet, temperature-controlled $\left(20-22^{\circ} \mathrm{C}\right)$ room between 10 a.m. and 4 p.m. PTZ-induced clonic seizure threshold was determined by inserting a 30-gauge needle into the tail vein of mice and infusion of $0.5 \%$ PTZ solution at a constant rate of $1 \mathrm{~mL} \mathrm{~min}^{-1}$ to unrestrained freely moving animals. Minimal dose of PTZ $\left(\mathrm{mg} \mathrm{kg}^{-1}\right.$ ) needed to induce forelimb clonus followed by full clonus of the body was recorded as an index of clonic seizure threshold (Samini et al., 2005; Shafaroodi et al., 2004).

Data analysis: Group data are presented as mean $\pm \mathrm{SEM}$ and analyzed statistically using Student test. Time course data were analyzed using one-way ANOVA followed by Tukey's post hoc test. The level for statistical significance was set at a $\mathrm{p}<0.05$.

\section{RESULTS AND DISCUSSION}

PTZ-induced seizure threshold in control mice was $35.48 \pm 1.39 \mathrm{mg} \mathrm{kg}^{-1}$. In vehicle group, $\mathrm{CMC}$ have not shown significant change on PTZ-induced seizure threshold compared with control group (Fig. 1).

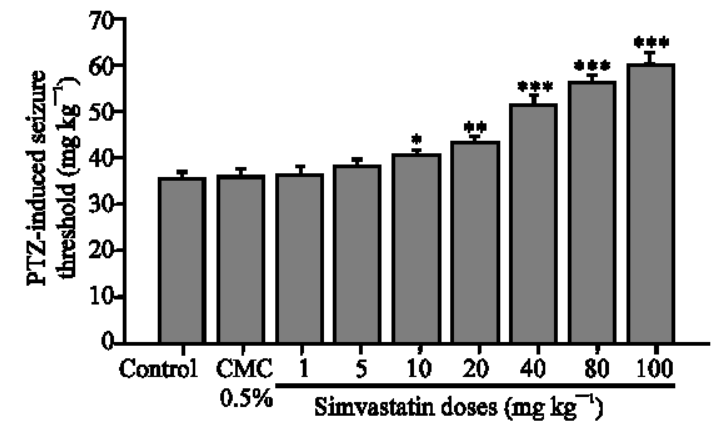

Fig. 1: PTZ-induced seizure threshold in mice $\left(\mathrm{mg} \mathrm{kg}^{-1}\right)$. Effect of intraperitoneally injection of different doses of simvastatin on seizure threshold. Each column represents mean \pm SEM of 8 mice. ${ }^{*} \mathrm{p}<0.05$, ${ }^{* *} \mathrm{p}<0.01$ and $* * * \mathrm{p}<0.001$ compared with control and vehicle groups
Intraperitoneal injection of simvastatin showed significant $(\mathrm{p}<0.05)$ increase of PTZ-induced seizure threshold in a dose dependently manner compared with control and vehicle groups (Fig. 1).

Pentylenetetrazole (PTZ) has been used widely to produce the animal model of chemically induced seizure because this model is highly sensitivity for comparing different chemical under standardized conditions (Samini et al., 2005; Shafaroodi et al., 2004). In this study, PTZ-induced seizure threshold in control mice was $35.48 \pm 1.39 \mathrm{mg} \mathrm{kg}^{-1}$ and simvastatin increased PTZ-induced seizure threshold in a dose dependently manner. Anti-nociceptive and anti-inflammatory effects of statins have been shown in different experimental animal models of pain and inflammatory and clinical studies (Dantas et al., 2010; Massaro et al., 2010; Nezic et al., 2009; Sparrow et al., 2001; Weitz-Schmidt et al., 2001) but so fare effects of statins on epilepsy are very limited (Lee et al., 2008; Rangel et al., 2005). Results of this study showed that simvastatin has beneficial neuroprotective effects on PTZ seizure model in mice that in agreement with a recent study that reported an effective neuroprotective action of statins after an acute brain injury and epilepsy (Daimona et al., 2004; Lee et al., 2008; Rangel et al., 2005). Furthermore, recent studies have shown that lovastatin partially suppressed acute Experimental Autoimmune Encephalomyelitis (EAE) in rat spinal cord injury model (Stanislaus et al., 1999). Also, studies reported that statin treatment provided anti-inflammatory effects on human multiple sclerosis and X-linked adrenoleukodystrophy (Nath et al., 2004; Paintlia et al., 2005; Pannu et al., 2007; Singh et al., 1998; Stanislaus et al., 1999, 2001, 2002; Vollmer et al., 2004). Moreover, other research groups have reported the similar neuroprotective role of statins in other neurological disease models such as traumatic brain injury, brain is chemia and Alzheimer's disease (Endres et al., 1998; Kirsch et al., 2003; Lu et al., 2004).

Rangel et al. (2005) demonstrated that lovastatin treatment was able to prevent hippocampal neuronal loss in CA1 subfield after an epileptic insult (Rangel et al., 2005). The molecular mechanisms of the anti-convulsant and immunomodulatory properties of statins have not been fully clarified. Rangel et al. (2005) suggested that Reactive Oxygen Species (ROS) are a part of normal human metabolism however when produced in excess, ROS can cause tissue injury including lipid peroxidation, DNA damage and enzyme inactivation. ROS is a common denominator among acute neurological conditions including epilepsy. In the Pilocarpine Model, there is an involvement of excitotoxic neuronal injury and ROS production has been considered to be a part of 
mechanisms involved with glutamatergic excitotoxicity in vitro and in vivo. Moreover, it was demonstrated that lovastatin treatment inhibits free radical injury.

Thus, this antioxidant effect of statins could explain the neuroprotective properties found in their study (Rangel et al., 2005). Also, they had been suggested that there is one classical argument supporting a possible role of Nitric Oxide (NO) in convulsive phenomena: excitatory amino acids such as N-Methyl-D-Aspartate (NMDA) and kainate are known to be potent convulsants and the activation of NMDA receptors is accompanied by the formation of NO. In fact, the role of NO in epileptogenesis has been examined in a number of studies suggesting to be a proconvulsive endogenous substance. Furthermore, some studies have revealed that statins inhibit the production of NO in brain parenchyma indicating that statins, secondarily may play the role of an anticonvulsant substance does not promoting glutamatemediated neurotoxicity. Other possibility is that the inhibition of brain endothelial Nitric Oxide Synthase (eNOS) leads to increased blood pressure which in turn may affect the excitability of central nervous system, however it was demonstrated that statins are able to upregulate eNOS may be pivotal in enhance cerebral arterial vasodilator responses, decreasing with this the firing threshold (Rangel et al., 2005).

Also, they had been suggested that antiinflammatory effects of statins could also contribute to neuroprotection after pilocarpine-induced status epilepticus. Moreover, several studies have implicated a number of cytokines in seizure-related pathology. Therefore, lovastatin treatment may provide an important approach to suppression of the inflammatory responses after status epilepticus (Rangel et al., 2005). In other study, Lee et al. (2008) reported that statins are inhibitors of HMG-CoA reductase, a rate limiting enzyme for synthesis of cholesterol and isoprenoids, thus their biological roles are expected to be mediated by their inhibitory roles in cholesterol and isoprenoid synthesis. Indeed, the inhibitory role of statins in cholesterol biosynthesis previously reported to inhibit NMDA mediated excitotoxicity in vitro neuron culture study.

Also, has been reported that statin treatment of cultured neuron cells leads to instability of AMPA receptor by disruption of cholesterol and sphingolipid rich membrane microdomains called Detergent Resistant/Insoluble Membrane Domains (DRM/DIM) or lipid rafts. Therefore, they had suggested that the possible involvement of cholesterol biosynthesis in statin-mediated anti-excitotoxic activity (Lee et al., 2008). At present whether cholesterol biosynthesis plays a role in statin-mediated anti-seizure and anti-excitotoxicity in the in vivo animal model is not known. In the brain, cholesterol has a very long half life (up to several months). Therefore, statins are not expected to change brain cholesterol level readily.

Previous studies have reported that systemic statin treatment does not affect cholesterol levels in the brain of guinea pigs. Nevertheless, previously reported reduction in cholesterol levels in lipid raft fractions of synaptosomal plasma membrane suggests that statin treatment may affect intercellular or intracellular distribution of cholesterol which is associated with glutamate receptor function in induction of status epilepticus and excitotoxicity (Lee et al., 2008). Along with cholesterol isoprenoids including farnesyl-pyrophosphate and geranylgeranylpyrophosphate are lipid byproducts produced by the mevalonate pathway and implicated in membrane attachment and function of small GTPases. Since, the small GTPases (i.e., RhoA and H-Ras) are involved in various neuronal functions and excitotoxic signaling cascades, statins are also expected to exert their anti-seizure and anti-excitotoxic activities through inhibition of isoprenoid synthesis and interfering with small GTPase signaling. Recent in vivo reports have demonstrated that inhibition of H-Ras farnesylation by Franeyltransferase Inhibitor (FTI) treatment inhibits NMDA-mediated excitotoxicity in the rat brain. This report supports that statins may modulate KA-mediated seizure activity and excitotoxicity by down-regulation of H-Ras isoprenylation.

However, whether statins exert their anti-seizure and anti-excitotoxic roles through inhibiting synthesis of isoprenoid or cholesterol or both of them is not known at present (Lee et al., 2008). Also, Lee et al. (2008) reported that neuroprotective efficacies of statins are mediated by their anti-inflammatory activity under various neurological disease conditions such as human multiple sclerosis and its animal models, spinal cord injury, X-linked adrenoleukodystrophy, Alzheimer's disease, traumatic brain injury and stroke. In these studies, statins inhibited inflammatory signal cascades and gene expression through inhibition of isoprenylation of small GTPase (Ras and RhoA).

A similar inhibitory role of statins in inflammatory reactions was observed in KA-treated rats. However, they do not expect that the observed statin-mediated reduction in inflammatory reactions as well as apoptotic cell death in KA-treated rat is mediated by its own anti-inflammatory activity observed in their previous studies. Since, post-seizure inflammation and leakage of blood-brain barrier are known to directly correlate with activity and frequency of status epilepticus, the decreased status epilepticus by statin treatment appears to attenuate 
inflammatory reaction in KA-treated rats (Lee et al., 2008). Statins have been used to reduce inflammation, tame immune cell activation or arrest degenerative processes. Because of their widespread use and long-term safety record some physicians prescribe statin therapy for non approved indications. A number of case reports describe the dramatic effects of statins added to standard therapies but beneficial effects need to be confirmed in controlled studies (Dinarello, 2010). Nevertheless, new and completely studies must be carried out in order to determine in more detail the beneficial actions of statins regarding the reduction of epilepsy.

\section{CONCLUSION}

According to researchers results, simvastatin has anticonvulsant effects on mice. Nevertheless, new studies must be carried out in order to determine the beneficial effects of statins in treatment of epilepsy.

\section{REFERENCES}

Daimona, M., S. Aomia, T. Kawamatab and H. Kurosawa, 2004. Pravastatin, a 3-hydroxy-3-methylglutaryl coenzyme A reductase inhibitor, reduces delayed neuronal death following transient forebrain ischemia in the adult rat hippocampus. Neurosci. Lett., 362: 122-126.

Dantas, A.C., L.F. Macedo, M.N. Mendes, I.M. Azevedo and A.C. Medeiros, 2010. Protective effect of simvastatin in the cyclophosphamide-induced hemorrhagic cystitis in rats. Acta Cir Bras., 25: 43-46.

Dinarello, C.A., 2010. Anti-inflammatory agents: Present and future. Cell, 140: 935-950.

Endres, M., U. Laufs, Z. Huang, T. Nakamura, P. Huang, M.A. Moskowitz and J.K. Liao, 1998. Stroke protection by 3-hydroxy-3-methylglutaryl (HMG)CoA reductase inhibitors mediated by endothelial nitric oxide synthase. Proc. Nat. Acad. Sci., 95: 8880-8885.

Goncalves, D.O., I.B.F. Calou, R.P. Siqueira, A.A. Lopes and L.K.A. Leal et al., 2011. In vivo and in vitro anti-inflammatory and anti-nociceptive activities of lovastatin in rodents. Braz. J. Med. Biol. Res., 44: 173-181.

Kirsch, C., G.P. Eckert, A.R. Koudinov and W.E. Muller, 2003. Brain cholesterol, statins and alzheimer's disease. Pharmacopsychiatry, 36: S113-S119.

Lee, J.K., J.S. Won, A.K. Singh and I. Singh, 2008. Statin inhibits kainic acid-induced seizure and associated inflammation and hippocampal cell death. Neurosci. Lett., 440: 260-264.
Lu, D., A. Goussev, J. Chen, P. Pannu, Y. Li, A. Mahmood and M. Chopp, 2004. Atorvastatin reduces neurological deficit and increases synaptogenesis, angiogenesis and neuronal survival in rats subjected to traumatic brain injury. J. Neurotrauma, 21: 21-32.

Massaro, M., A. Zampolli, E. Scoditti, M.A. Carluccio, C. Storelli, A. Distante and R. De Caterina, 2010. Statins inhibit cyclooxygenase-2 and matrix metalloproteinase-9 in human endothelial cells: Antiangiogenic actions possibly contributing to plaque stability. Cardiovasc. Res., 86: 311-320.

McCarey, D.W., I. McInnes, R. Mahdhok, R. Hampson and O. Scherbakova et al., 2004. Trial of Atorvastatin in Rheumatoid Arthritis (TARA): Double-blind, randomised placebo-controlled trial. Lancet, 363: 2015-2021.

McTaggart, F., L. Buckett, R. Davidson, G. Holdgate and A. McCormick et al., 2001. Preclinical and clinical pharmacology of Rosuvastatin, a new 3-hydroxy-3methylglutaryl coenzyme a reductase inhibitor. Am. J. Cardiol., 87: 28B-32B.

Nath, N., S. Giri, R. Prasad, A.K. Singh and I. Singh, 2004. Potential targets of 3-hydroxy-3-methylglutaryl coenzyme A reductase inhibitor for multiple sclerosis therapy. J. Immunol., 172: 1273-1286.

Nezic, L., R. Skrbic, S. Dobric, M.P. Stojiljkovic, S.S. Satara, Z.A. Milovanovic and N. Stojakovic, 2009. Effect of simvastatin on proinflammatory cytokines production during lipopolysaccharideinduced inflammation in rats. Gen Physiol. Biophys., 28: 119-126.

Paintlia, A.S., M.K. Paintlia, M. Khan, T. Vollmer, A.K. Singh and I. Singh, 2005. HMG-CoA reductase inhibitor augments survival and differentiation of oligodendrocyte progenitors in animal model of multiple sclerosis. Fed. Am. Soc. Exp. Biol. J., 19: 1407-1421.

Pannu, R., D.K. Christie, E. Barbosa, I. Singh and A.K. Singh, 2007. Post-trauma lipitor treatment prevents endothelial dysfunction, facilitates neuroprotection and promotes locomotor recovery following spinal cord injury. J. Neurochem., 101: 182-200.

Rangel, P., R.M. Cysneiros, R.M. Arida, M. de Albuquerque and D.B. Colugnati et al., 2005. Lovastatin reduced neuronal cell death in hippocampal CA1 subfield after pilocarpine-induced status epilepticus: Preliminary results. Arq Neuropsiquiatr., 63: 972-976.

Samini, M., H. Shafaroodi, V. Sehat and S. Allahdini, 2005. Comparison of the effects of lindane and permethrin on pentylenetetrazole-induced clonic seizure in mice. Online J. Vet. Res., 9: 37-47. 
Scandinavian Simvastatin Survival Study, 1994. Randomised trial of cholesterol lowering in 4444 patients with coronary heart disease: The Scandinavian Simvastatin Survival Study (4S). Lancet, 344: 1383-1389.

Shafaroodi, H., M. Samini, L. Moezi, H. Homayoun and H. Sadeghipour et al., 2004. The interaction of cannabinoids and opioids on pentylenetetrazoleinduced seizure threshold in mice. Neuropharmacology, 47: 390-400.

Shepherd, J., S.M. Cobbe, I. Ford, C.G. Isles and A.R. Lorimer et al., 1995. Prevention of coronary heart disease with pravastatin in men with hypercholesterolemia. West of Scotland Coronary Prevention Study Group. N. Engl. J. Med., 333: 1301-1307.

Singh, I., K. Pahan and M. Khan, 1998. Lovastatin and sodium phenylacetate normalize the levels of very long chain fatty acids in skin fibroblasts of Xadrenoleukodystrophy. FEBS Lett., 426: 342-346.

Sironi, L., M. Cinino, U. Guerrini, A.M. Calvio and B. Lodetti et al., 2003. Treatment with statins after induction of focal ischemia in rats reduces the extent of brain damage. Arterioscler. Thromb. Vasc. Biol., 23: $322-327$.
Sparrow, C.P., C.A. Burton, M. Hernandez, S. Mundt and H. Hassing et al., 2001. Simvastatin has antiinflammatory and antiatherosclerotic activities independent of plasma cholesterol lowering. Arterioscler. Thromb. Vasc. Biol., 21: 115-115.

Stanislaus, R., K. Pahan, A.K. Singh and I. Singh, 1999. Amelioration of experimental allergic encephalomyelitis in Lewis rats by lovastatin. Neurosci. Lett., 269: 71-74.

Stanislaus, R., A.K. Singh and I. Singh, 2001. Lovastatin treatment decreases mononuclear cell infiltration into the CNS of Lewis rats with experimental allergic encephalomyelitis. J. Neurosci. Res., 66: 155-162.

Stanislaus, R., A.G. Gilg, A.K. Singh and I. Singh, 2002. Immunomodulation of experimental autoimmune encephalomyelitis in the Lewis rats by Lovastatin. Neurosci. Lett., 333: 167-170.

Vollmer, T., L. Key, V. Durkalski, W. Tyor and J. Corboy, 2004. Oral simvastatin treatment in relapsing-remitting multiple sclerosis. Lancet, 363: 1607-1608.

Wang, C.Y., P. Liu and J.K. Liao, 2008. Pleiotropic effects of statin therapy: Molecular mechanisms and clinical results. Trends Mol. Med., 14: 37-44.

Weitz-Schmidt, G., K. Welzenbach, V. Brinkmann, T. Kamata and J. Kallen et al., 2001. Statins selectively inhibit leukocyte function antigen-1 by binding to a novel regulatory integrin site. Nat. Med., 7: $687-692$. 